\title{
Doppler frequency offset estimation and diversity reception scheme of high-speed railway with multiple antennas on separated carriage
}

\author{
Yaoqing YANG $^{1}$, Pingyi FAN ${ }^{1,2 *}$ \\ 1. Department of Electronic Engineering, Tsinghua University, Beijing 100084, China \\ 2. National Mobile Communications Research Laboratory, Southeast University, Nanjing 210096, China
}

\begin{abstract}
The challenges of severe Doppler effects in high-speed railway are considered. By building a cooperative antenna system, an algorithm of joint channel estimation and Doppler frequency offset (DFO) estimation is proposed based on Ricean channel model. First, a maximum likelihood estimation (MLE) algorithm for DFO is designed, showing that the Doppler estimation can be obtained by estimating moving velocity of the train and the path loss with the exploitation of pilots that are placed inside the frame. Then a joint detection algorithm for the receiver is proposed to exploit multi-antenna diversity gains. Last, the theoretical Crammer Rao bound (CRB) for joint channel estimation and DFO estimation is derived. The steady performance of the system is confirmed by numerical simulations. In particular, when the Ricean fading channel parameter equals 5 and the velocities of train are $100 \mathrm{~m} / \mathrm{s}$ and $150 \mathrm{~m} / \mathrm{s}$, the estimation variances of DFO are very close to the theoretical results obtained by using CRB. Meanwhile, the corresponding signal to noise ratio loss is less than $1.5 \mathrm{~dB}$ when the bit error rate is $10^{-5}$ for 16QAM signals.
\end{abstract}

Key words: Doppler frequency offset (DFO); high-speed railway; Ricean channel; cooperative antenna system

(C) 2012 JMT. All rights reserved.

\section{Introduction}

$\mathrm{O}$ ne of the challenges in the fast development of high-speed railway (HSR) in China is that current systems, such as GSM-R which only supports a maximum data rate of $200 \mathrm{kbps}$ [1], cannot meet the increasing demands for multi-media access while traveling. Many efforts have been made to design an advanced communication system for extreme environmental conditions, and to develop the overall working capabilities of newly proposed standards such as LTE-R and WiMAX [2-3].

The most challenging problem in HSR is the severe Doppler effects caused by high speeds up to $481.6 \mathrm{~km} / \mathrm{h}$ [4]. Hence evaluating Doppler spread so as to remove frequency offset becomes crucial [5]. Most of current work on Doppler estimation emphasizes the direct estimate of Doppler frequency offset (DFO) in OFDM systems [6-7]. It was reported recently that multi-path effects in HSR channels are much great, due

Received Oct. 21, 2012; revision accepted Nov. 24, 2012

${ }^{*}$ Corresponding author. E-mail: fpy@mail.tsinghua.edu.cn (P.Y. FAN)

(C) 2012 JMT. All rights reserved

doi: 10.3969/j.issn.2095-087X.2012.04.006 to the dominant existence of line-of-sight (LOS) components $[4,7]$. Thus it is important to first specify the channel models, and then design algorithms to estimate Doppler effects. This paper, based on the Ricean channel model, investigates the effect of LOS component and the path loss. Also, power issues are highlighted since the signals may suffer from severe penetration loss when going through the carriage body made of aluminum and stainless steel [4]. Many antenna-based technologies have been proposed to solve this problem. In Ref. [3], a promising solution was proposed by using a receive antenna outside the train body to communicate with a group of base stations. This technology greatly reduces penetration loss. In this paper, the technology is extended to multi-antenna situations, in the case that there is one receive antenna on each carriage of the train. This extension has proved to be effective in improving the performance of Doppler frequency offset estimation.

The main contribution of this work is to build a cooperative antenna system outside the train body and design joint channel estimation and Doppler frequency offset (DFO) estimation algorithm. Furthermore, the theoretical Crammer Rao bound (CRB) for estimation is derived based on Ricean channel model. Finally, the performance of the proposed system is investigated by simulation. 


\section{System model}

\subsection{Cooperative antenna system for HSR}

Suppose that there is a fast moving train. It will receive the downlink signal from a base station. The train has a cooperative multi-antenna receiving system with one receive antenna on each carriages, as shown in Fig. 1. The base station is set close to the rail with a distance of $d$. The typical value for $d$ is about $30 \mathrm{~m} \mathrm{[4].}$ Since base station has a height, we define $d=50 \mathrm{~m}$ in simulation section. The foot point is defined as point $O$, and the distance between point $O$ and the first receive antenna is $a$.

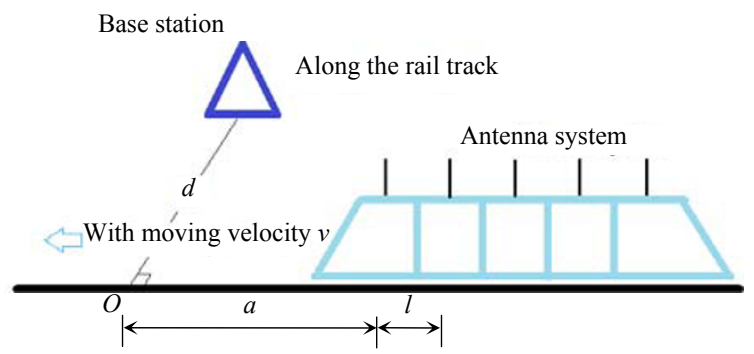

Fig. 1 A cooperative antenna system for HSR

The train is moving toward $O$ on the rail with the velocity $v$; so the Doppler shift for the first antenna caused by high moving velocity can be defined as

$$
\begin{aligned}
& D(t)=\frac{f_{c} v}{c} \cos \theta(t), \\
& \cos \theta(t)=\frac{a-v t}{\sqrt{(a-v t)^{2}+d^{2}}} .
\end{aligned}
$$

One receiving antenna was installed on each carriage of the train. The length of the train carriage is $l$, so is the separation distance between two neighboring receive antennas. Typical value for $l$ is about $10 \mathrm{~m}$, long enough for the additional noise resulting from each antenna to be irrelevant. The antennas are numbered from $n=0$ to $N$, and the Doppler shift for each antenna can be defined as

$$
\begin{aligned}
& D_{n}(t)=\frac{f_{c} v}{c} \cos \theta_{n}(t), \\
& \cos \theta_{n}(t)=\frac{a+n l-v t}{\sqrt{(a+n l-v t)^{2}+d^{2}}}, n=0 \sim N .
\end{aligned}
$$

\subsection{Channel modeling}

The base station modulates the transmitted signal $s(t)$ with a carrier frequency $f_{c}$. The received signal $x(t)$ suffers from a power loss $1 / l(t)^{\alpha}$, a frequency shift $D$ and an added white Gaussian noise $w(t)$ with a variance of $2 \sigma^{2}$. The random phase shift $\phi$ caused by transmission is evenly distributed in $[0,2 \pi)$. Then the received signal $x(t)$ can be written as

$$
x(t)=\frac{s(t) \exp \left(\mathrm{j} 2 \pi\left(f_{c}+D\right) t+\phi\right)}{l(t)^{\alpha}}+w(t) .
$$

All the $N$ antennas can receive signals, and we sample the baseband received signal with a sampling period $T_{s}$. Then, the received signal can be expressed as

$$
\begin{gathered}
x_{n}(m)=s(m) \frac{\exp \left(\mathrm{j}\left[2 \pi D_{n} m T_{s}+\phi_{n}\right]\right)}{l_{n}{ }^{\alpha}}+w_{n}(m), \\
n=0,1 \cdots, N ; \quad m=0,1, \cdots, M-1,
\end{gathered}
$$

where $l_{n}=a+n l, D_{n}$ is defined in (3) and $M$ represents the number of symbols in a data frame. In this simulation, we define $N=16$, meaning that one train has 17 carriages.

Then we slightly modify the channel representation in (6) by introducing Ricean fading effect:

$$
x_{n}(m)=s(m) \frac{h_{n}}{l_{n}^{\alpha}} \exp \left(\mathrm{j} 2 \pi D_{n} m T_{s}\right)+w_{n}(m),
$$

where

$$
h_{n}=\sqrt{\frac{\kappa}{\kappa+1}} \exp \left(\mathrm{j} \varphi_{n}\right)+\sqrt{\frac{1}{\kappa+1}} z_{n}=r_{n} \exp \left(\mathrm{j} \phi_{n}\right)
$$

and $z_{n} \sim C N(0,1), \kappa$ is the so-called Ricean factor, $r_{n}$ obeys Ricean distribution with the center 1 , and $\phi_{n}$ is evenly distributed in $[0,2 \pi)$, similar to the definition in (6).

The probability density function (PDF) of all $r_{n}$ can be written as

$$
f(r)=2(k+1) r \exp \left(-\frac{r^{2}+k /(k+1)}{k /(k+1)}\right) I_{0}(2 \sqrt{k(k+1)} r),
$$

where $I_{0}(\cdot)$ is the zero order Bessel function.

\subsection{Frame configuration and pilot position}

Estimating the Doppler shift is done by inserting pilots into a data frame. As is shown in (7), Doppler shift brings a change in the phase of each received symbol. In order to get enough information for Doppler frequency offset estimation, pilots are inserted into separated places of a frame, as shown in Fig. 2. We put $K$ pilots into $M$ receive symbols to have $(M-K) / K$ symbols after each pilot. The ratio $(M-K) / K$ can represent the effi- 
ciency of transmission. There is certainly a trade-off between estimation accuracy and transmission efficiency as well as estimation complexity.

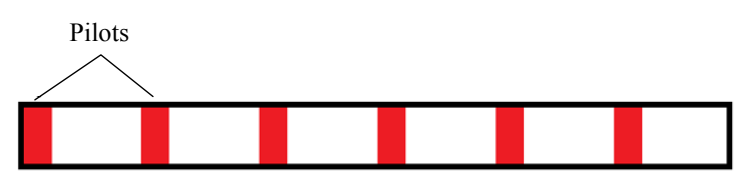

Fig. 2 Frame configuration and pilot position

When the train speed is $100 \mathrm{~m} / \mathrm{s}$ and $f_{c}=1.8 \mathrm{GHz}$, the coherent time will be $1 / D=c / f v \approx 1.6 \mathrm{~ms}$, and we can set a frame interval to $1 \mathrm{~ms}$ to minimize the effects of Doppler frequency shift.

\section{Joint DFO and channel estimation}

For each frame, $K$ pilots are used to estimate the Doppler frequency shift. It must be stated here that a frame needs to be designed properly to make sure that the Doppler frequency shift will not change in one frame. The ideal frame length should be less than $1 / D_{\max }$, where $D_{\max }$ represents the maximum Doppler shift.

With considering antenna $n$, the whole received signals $x_{n}(k)$ for pilots $s(k)$ are represented as

$$
\begin{gathered}
x_{n}(k)=s(k) \frac{h_{n}}{l_{n}^{\alpha}} \exp \left(\mathrm{j} 2 \pi D_{n} k \frac{M}{K} T_{s}\right)+w_{n}(k), \\
n=0,1, \cdots, N, \quad k=0,1, \cdots, K-1,
\end{gathered}
$$

where all $h_{n}$ are defined in (8).

Define

$$
A_{n}=\frac{r_{n}}{l_{n}{ }^{\alpha}}, B_{n}(k)=2 \pi D_{n} k \frac{M}{K} T_{s},
$$

and simply take $s(k)=1$ for each pilot. Then Eq. (10) can be simplified into

$$
\begin{gathered}
x_{n}(k)=A_{n} \exp \left\{\mathrm{j}\left[B_{n}(k)+\phi_{n}\right]\right\}+w_{n}(k), \\
n=0,1, \cdots, N, k=0,1, \cdots, K-1 .
\end{gathered}
$$

All $D_{n}$ are determined by Eqs. (3) and (4) and the distance $a$ is estimated with cutting-edge equipments, such as a laser ranging equipment. The estimation of velocity $v$ and channel fading coefficients $h_{n}$ need to be investigated. However, the velocity estimation remains a tough problem for mobile communication [8-10]. In this paper we propose a maximum likelihood estimation (MLE) algorithm to estimate the velocity. If the two parameters $a$ and $v$ are determined, we can calculate each $D_{n}$ directly.
With the assumption that $w_{n}(k)$ is additive white Guassian noise (AWGN), the PDF for each $x_{n}(k)$ is

$$
\begin{aligned}
& f\left(x_{n}(k) ; v, h\right)=\frac{1}{(\sqrt{2 \pi} \sigma)^{2}} \exp \left\{\frac { - 1 } { 2 \sigma ^ { 2 } } \left[x_{n}^{R}(k)-\right.\right. \\
& \left.\left.A_{n} \cos \left(B_{n}(k)+\phi_{n}\right)\right]^{2}\right\} \exp \left\{\frac { - 1 } { 2 \sigma ^ { 2 } } \left[x_{n}^{I}(k)-\right.\right. \\
& \left.\left.A_{n} \sin \left(B_{n}(k)+\phi_{n}\right)\right]^{2}\right\},
\end{aligned}
$$

where $x_{n}^{R}(k)$ and $x_{n}^{I}(k)$ are real and imaginary parts of $x_{n}(k)$, respectively, and

$$
f(\boldsymbol{x} ; v, \boldsymbol{h})=\prod_{n=0}^{N} \prod_{k=1}^{K} f\left(x_{n}(k) ; v, h\right) .
$$

\subsection{MAP estimation for path loss $r_{n}$}

We focus on the path loss estimation for each $r_{n_{0}}$. Eq. (14) is slightly different from those deduced in previous works [8-9] in that we use MAP (maximum a posteriori) estimation instead of the ML (maximum likelihood) estimation in order for better performance based on the prior PDF of $r_{n_{0}}$ in (9).

Hence, one must solve the following equation:

$$
\begin{aligned}
0= & \frac{\partial}{\partial r_{n_{0}}} \ln f(x ; h, v) f(h)= \\
& \frac{\partial}{\partial r_{n_{0}}} \ln \left[\prod_{n=0}^{N} \prod_{k=1}^{K} f\left(x_{n}(k) ; h, v\right)\right]+\frac{\partial}{\partial r_{n_{0}}} \ln f(h)= \\
& \frac{\partial}{\partial r_{n_{0}}} \ln f\left(r_{n_{0}}\right)+\frac{1}{\sigma^{2}} \times \\
& \sum_{k=1}^{K} \frac{1}{l_{n_{0}}^{\alpha}} \cos \left(B_{n_{0}}(k)+\phi_{n_{0}}\right)\left(x_{n_{0}}^{R}(k)-A_{n_{0}} \cos \left(B_{n_{0}}(k)+\phi_{n_{0}}\right)\right)+ \\
& \frac{1}{l_{n_{0}}^{\alpha}} \sin \left(B_{n_{0}}(k)+\phi_{n_{0}}\right)\left(x_{n_{0}}^{I}(k)-A_{n_{0}} \sin \left(B_{n_{0}}(k)+\phi_{n_{0}}\right)\right) .
\end{aligned}
$$

Since $x_{n}^{R}(k)$ is the real part of $x_{n}(k)$ shown in (12), it is clear that

$$
x_{n}^{R}(k) \cos \left(B_{n}(k)+\phi_{n}\right)+x_{n}^{I}(k) \sin \left(B_{n}(k)+\phi_{n}\right) \approx\left|x_{n}(k)\right| .
$$

The equation above can be simplified to

$$
\frac{1}{\sigma^{2}}\left[\sum_{k=1}^{K} \frac{1}{l_{n}^{\alpha}}\left|x_{n}(k)\right|-\frac{r_{n_{0}}}{\left|l_{n}^{\alpha}\right|^{2}}\right]+\frac{\partial \ln f_{R}\left(r_{n_{0}}\right)}{\partial r}=0,
$$

or 


$$
\begin{gathered}
r_{n_{0}}=\operatorname{argmax} \frac{1}{\sigma^{2}}\left(\sum_{k=1}^{K} \frac{1}{l_{n}^{\alpha}}\left|x_{n}(k)\right|\right) r- \\
\frac{1}{2}\left(\sum_{k=1}^{K} \frac{1}{\left|l_{n}^{\alpha}\right|^{2}}\right) r^{2}+f_{R}(r) .
\end{gathered}
$$

\subsection{ML estimation for velocity $v$}

For a better estimation of Doppler shift, the estimation for velocity needs to be solved. To solve $\partial f(x, v, h) / \partial v=0$, we have

$$
\begin{aligned}
\hat{v} & =\operatorname{argmax} \frac{-1}{\sigma^{2}} \sum_{n=0}^{N} \sum_{k=1}^{K}\left[\left[x_{n}^{R}(k)-A_{n} \cos \left(B_{n}(k)+\phi_{n}\right)\right]^{2}-\right. \\
& {\left.\left[x_{n}^{I}(k)-A_{n} \sin \left(B_{n}(k)+\phi_{n}\right)\right]^{2}\right]=} \\
& \operatorname{argmax} \sum_{n=0}^{N} \sum_{k=1}^{K} x_{n}^{R}(k) A_{n} \cos \left(B_{n}(k)+\phi_{n}\right)+ \\
& x_{n}^{I}(k) A_{n} \sin \left(B_{n}(k)+\phi_{n}\right) \\
& =\operatorname{argmax} \operatorname{Re}\left[\sum_{n=0}^{N} A_{n} \sum_{k=1}^{K} x_{n}(k) \exp \left\{-\mathrm{j}\left(B_{n}(k)+\phi_{n}\right)\right\}\right] \\
& \approx \operatorname{argmax} \operatorname{abs}\left[\sum_{n=0}^{N} A_{n} \sum_{k=1}^{K} x_{n}(k) \exp \left(-\mathrm{j} B_{n}(k)\right)\right] .
\end{aligned}
$$

\subsection{ML estimation for random phase}

To obtain the phase estimation, one need to solve

$$
\begin{aligned}
0= & \frac{\partial}{\partial \phi_{n_{0}}} \ln f(\vec{x} ; h, v) f(h)= \\
& \frac{\partial}{\partial \phi_{n_{0}}} \ln \left[\prod_{n=0}^{N} \prod_{k=1}^{K} f\left(x_{n}^{R}(k) ; h, v\right)\right]+\frac{\partial}{\partial \phi_{n_{0}}} \ln f(h)= \\
& \frac{A_{n_{0}}}{\sigma^{2}} \sum_{k=1}^{K}\left[-\sin \left(B_{n_{0}}(k)+\phi_{n_{0}}\right)\right] x_{n_{0}}^{R}(k)+ \\
& \frac{1}{l_{n_{0}}{ }^{\alpha}} \cos \left(B_{n_{0}}(k)+\phi_{n_{0}}\right) x_{n_{0}}^{R}(k) .
\end{aligned}
$$

which means

$$
\begin{aligned}
\hat{\phi}_{n_{0}} & =\operatorname{argmax} \frac{1}{\sigma^{2}} \sum_{k=1}^{K}\left[A_{n_{0}} \cos \left(B_{n_{0}}(k)+\phi_{n_{0}}\right) x_{n_{0}}^{R}(k)+\right. \\
& \left.A_{n_{0}} \sin \left(B_{n_{0}}(k)+\phi_{n_{0}}\right) x_{n_{0}}^{I}(k)\right] \\
= & \operatorname{argmax} \frac{1}{\sigma^{2}} \sum_{k=1}^{K} \operatorname{Re}\left[x_{n}(k) \exp \left(-\mathrm{j}\left(B_{n_{0}}(k)+\phi_{n_{0}}\right)\right)\right] \\
= & \operatorname{argmax} \frac{1}{\sigma^{2}} \times \\
& \operatorname{Re}\left[\exp \left(-\mathrm{j} \phi_{n_{0}}\right) \sum_{k=1}^{K} x_{n}(k) \exp \left(-\mathrm{j} B_{n_{0}}(k)\right)\right] \\
= & \operatorname{angle}\left[\sum_{k=1}^{K} x_{n}(k) \exp \left(-\mathrm{j} B_{n_{0}}(k)\right)\right] .
\end{aligned}
$$

In summary, the whole estimation steps are listed:

(1) Estimate the path loss $r_{n}$ using (16).

(2) Estimate velocity $v$ using (17) and calculate the Doppler shift $D_{n}$.

(3) Estimate the random phase $\phi_{n}$ using (18).

After calculating all parameters, we can then decode the received signals in the frame. Simulation results will prove the efficiency of estimation algorithm.

\section{Theoretical symbol error rate (SER) per- formance}

The received signals can be written as (7). By defining

$$
h_{n}(m)=\frac{r_{n} \exp \left(\mathrm{j}\left(2 \pi D_{n} m T_{s}+\phi_{n}\right)\right)}{l_{n}^{\alpha}},
$$

we have

$$
\begin{aligned}
& x_{n}(m)=s(m) h_{n}(m)+w_{n}(m), \\
& \quad n=0,1, \cdots N, \quad m=0,1, \cdots, M-1 .
\end{aligned}
$$

Therefore, the linear combination of all the signals is given as follows:

$$
\begin{aligned}
& y(m)=\sum_{n=1}^{N} \gamma_{n}(m) x_{n}(m)= \\
& \sum_{n=1}^{N} \gamma_{n}(m) h_{n}(m) s(m)+\gamma_{n}(m) w_{n}(m) .
\end{aligned}
$$

The corresponding SNR for each symbol is

$$
\begin{aligned}
S N R & =\frac{\left|\sum_{n=1}^{N} \gamma_{n}(m) h_{n}(m) s(m)\right|^{2}}{\left|\gamma_{n}(m) w_{n}(m)\right|^{2}} \\
& =\frac{|s(m)|^{2}}{2 \sigma^{2}} \frac{\left|\sum_{n=1}^{N} \gamma_{n}(m) w_{n}(m)\right|^{2}}{\sum_{i=1}^{N}\left|\gamma_{n}(m)\right|^{2}} \\
& =\frac{|s(m)|^{2}}{2 \sigma^{2}} \frac{(\Gamma H)^{2}}{|\Gamma|^{2}} .
\end{aligned}
$$

If all the parameters are accurately estimated, we can simply let

$$
\Gamma=\frac{H^{*}}{|H|}
$$

to achieve the highest diversity gains and get

$$
S N R_{\max }=\frac{|s(m)|^{2}}{2 \sigma^{2}}|H|^{2} .
$$


From Ref. [11], we know that

$$
P(A \stackrel{\text { Error }}{\longrightarrow} B)=Q\left(\frac{\left\|\boldsymbol{u}_{A}-\boldsymbol{u}_{B}\right\| \cdot\|H\|}{2 \sigma}\right),
$$

where $\left\|\boldsymbol{u}_{A}-\boldsymbol{u}_{B}\right\|$ is the minimum distance on the constellation diagram. The $A$ and $B$ are two neighboring constellation points. In the simulation section, choosing 16QAM as the modulation scheme leads to a better spectrum efficiency. From Ref. [12] we have

$$
\begin{aligned}
& S N R_{\max }=E\left(\frac{|s(m)|^{2}}{2 \sigma^{2}}|H|^{2}\right)= \\
& \frac{25}{8}\left(\frac{\left\|\boldsymbol{u}_{A}-\boldsymbol{u}_{B}\right\| \cdot\|H\|}{2 \sigma}\right)^{2}, \\
& B E R=\frac{3}{4} Q\left(\sqrt{\frac{1}{10} S N R_{\max }}\right)=\frac{3}{4} Q\left(\sqrt{\frac{4}{5} \frac{E_{b}}{n_{0}}}\right) .
\end{aligned}
$$

\section{Crammer Rao bounds for estimation}

The exact information of $r_{n}$ and $\phi_{n}$ is unknown. When $r_{n}, \phi_{n}$ and $v$ are determined, each received symbol $x_{n}(k)$ obeys a complex normal distribution. The PDF of each $x_{n}(k)$ and the joint distribution of all $x_{n}(k)$ can be written as Eqs. (13) and (14).

CRBs can be found by calculating the Fisher information matrix first. The Fisher information content for each parameter is defined as

$$
I_{\theta}=E\left[\frac{\partial \ln f(\boldsymbol{x} ; \theta)}{\partial \theta}\right]^{2}, \quad \theta=v, r, \phi
$$

Then we have

$$
\begin{aligned}
& I_{\theta}=\frac{1}{\sigma^{2}}\left[\sum_{n=1}^{N} \sum_{k=1}^{M}\left(\frac{\partial \operatorname{Re}\left(s_{n} A_{n} \exp \left[\mathrm{j}\left(\phi_{n}+B_{n}(k)\right)\right]\right.}{\partial \theta}\right)^{2}+\right. \\
&\left.\sum_{n=1}^{N} \sum_{k=1}^{M}\left(\frac{\partial \operatorname{Im}\left(s_{n} A_{n} \exp \left[\mathrm{j}\left(\phi_{n}+B_{n}(k)\right)\right]\right.}{\partial \theta}\right)^{2}\right] \\
&=\frac{1}{\sigma^{2}} \sum_{n=1}^{N} \sum_{k=1}^{M}\left(A_{n}^{2} \frac{\partial\left(B_{n}(k)+\phi_{n}\right)}{\partial \theta}+\left(\frac{\partial A_{n}}{\partial \theta}\right)^{2}\right)^{2}, \\
& \theta=v, r, \phi .
\end{aligned}
$$

Similarly, the Fisher information content for joint parameters $I_{\theta_{1} \theta_{2}}$ is

$$
I_{\theta_{i} \theta_{j}}=\frac{1}{\sigma^{2}} \sum_{n=1}^{N} \sum_{k=1}^{M}\left[A_{n}^{2} \frac{\partial B_{n}(k)}{\partial \theta_{i}} \frac{\partial B_{n}(k)}{\partial \theta_{j}}+\frac{\partial A_{n}}{\partial \theta_{i}} \frac{\partial A_{n}}{\partial \theta_{j}}\right]
$$

Finally, the Fisher matrix is obtained

$$
\boldsymbol{I}=\left[I_{\theta_{i} \theta_{j}}\right],
$$

and the Crammer Rao Bounds can be calculated as

$$
C R B=I^{-1} .
$$

The diagonal components of matrix $\boldsymbol{C R B}$ are the real variance bounds for the estimated parameters. From (30), $I_{r_{n} \phi_{n}}=0$ and $I_{v r_{n}}=0$, because $A_{n}$ has nothing to do with $v$ and $\phi_{n}$, and $B_{n}(k)$ has nothing to do with $r_{n}$.

To measure the accuracy of Doppler offset estimation, we need to calculate the CRB for $v$; in other words, we only need to calculate Fisher Matrix for $v$ and all $\phi_{n}$, that is,

$$
\boldsymbol{I}=\left[I_{v}, \boldsymbol{I}_{v \phi} ; \boldsymbol{I}_{v \phi}^{\mathrm{T}}, \boldsymbol{I}_{\phi}\right],
$$

where $\boldsymbol{I}_{\phi}$ is a diagonal matrix, and $\boldsymbol{I}_{v \phi}$ is a row vector.

From (32), finally we can get CRB for $v$ :

$$
C R B_{v}=\boldsymbol{I}^{-1}(1,1)=1 /\left(I_{v}-\boldsymbol{I}_{v \phi} \boldsymbol{I}_{\phi}{ }^{-1} \boldsymbol{I}_{v \phi}{ }^{\mathrm{T}}\right) .
$$

To calculate the CRB for channel estimation, we have to calculate $C R B_{r_{n}}$ by (30) and $C R B_{\phi_{n}}$ from the inverse of (33).

From (8), we have

$$
C R B_{h_{n}}=C R B_{r_{n}}+r_{n}^{2} C R B_{\phi_{n}} .
$$

The closed form for CRB in (32) is redundant here, because $I_{\theta_{i} \theta_{j}}$ is represented by many summation forms. Note that $C R B_{v}$ is irrelevant to the velocity. Thus it is expected that the estimation can have a steady performance in different situations. However, the CRB for velocity will have some random variables in its expression. Here we choose the expectation of $C R B_{v}$ to represent its theoretical performance.

\section{Simulation}

Let $f_{c}=2.35 \mathrm{GHz}$, and the sampling time $T_{s}=$ $0.25 \mu \mathrm{s}$. The time range for one frame is $1 \mathrm{~ms}$. Each frame has $M=4000$ symbols, and $K=50$ symbols are used as pilots to estimate the Doppler shift.

Assume that a relatively accurate estimation of distance $a$ is reached with laser ranging equipment installed on the base station when the train is within $100 \mathrm{~m}$ of the base station. The estimation error is set to $1 \%$ of the real distance. When the train is out of this range, it can be assumed that a rough estimation is ensured with equipment like GPS and control estimation error to be no more than $5 \%$ of the whole distance. 


\subsection{Estimation performance compared with $C R B$}

Fig. 3 shows the relative Doppler frequency estimation error for the 9th antenna when $v=100 \mathrm{~m} / \mathrm{s}$ and $k=5$. In fact, it was reported the typical value for $k$ ranged between 5 and 10 and its average value for $k$ was about 8.25 [4]. The theoretical results are calculated according to the procedure in Section 5.

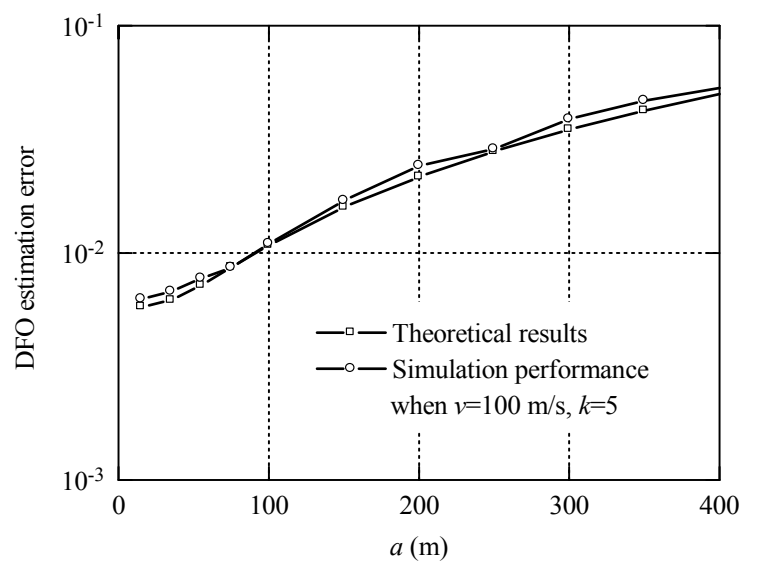

Fig. 3 Relative DFO estimation error performance when $v=100 \mathrm{~m} / \mathrm{s}$

Fig. 4 shows the situation when $v=150 \mathrm{~m} / \mathrm{s}$ and $k=5$, where the Doppler frequency offset increases a lot. With the speed increasing from 100 to $150 \mathrm{~m} / \mathrm{s}$, the absolute estimation error has no obvious increase, which means that the relative error drops. The simulation results also indicate that the performance of the proposed DFO estimation algorithm is stable, since the estimation error is very close to that obtained by using CRB. This is because that $\mathrm{CRB}$ is the lowest bound of all the unbiased parameter estimates.

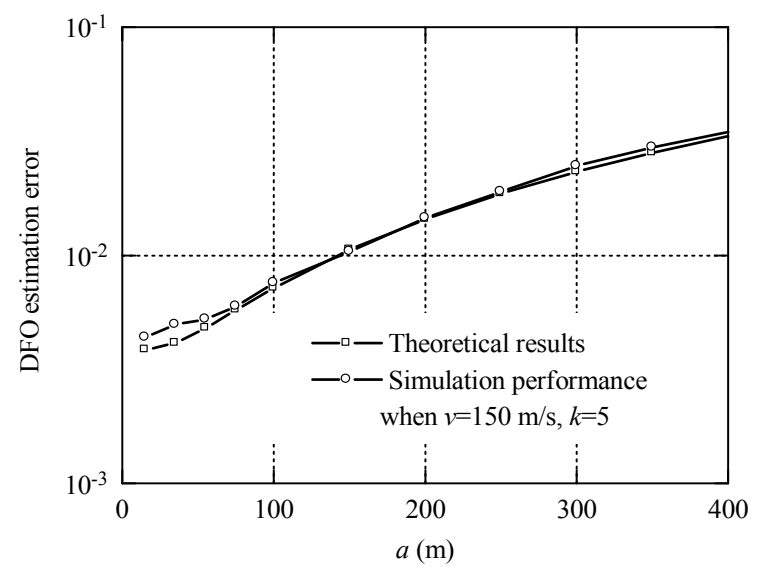

Fig. 4 Relative DFO estimation error performance when $v=150 \mathrm{~m} / \mathrm{s}$

\subsection{BER performance compared with AWGN channel}

Fig. 5 shows the bit error rate (BER) using 16QAM. The theoretical BER value is derived in Section 6 . This calculation is based on the AWGN channel when $\kappa=\infty$. There is still some loss in BER performance due to the inaccuracy of joint channel and DFO estimation. The BER performance becomes slightly better when $k=10$, since the channel fading becomes less obvious.

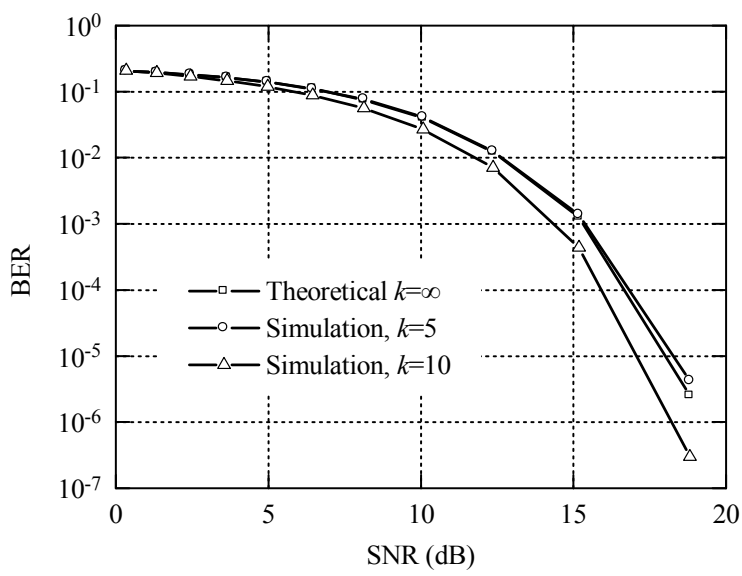

Fig. 5 BER performance comparison of 16QAM with and without DFO estimations for different Ricean channels

\section{Conclusions}

A cooperative antenna system is set up to solve the problems of severe Doppler effects emerging in highspeed railways, and an algorithm for joint channel estimation and Doppler frequency offset (DFO) estimation is proposed. Because the relative moving speeds of every receiving antennas outside the carriage are different, the DFOs for every antennas are also different. In order to improve the DFO estimation performance by exploiting the benefit of multi-antenna diversity, a new scheme to estimate the Doppler frequency offsets is developed. The theoretical deduction shows that the Doppler estimation can be obtained by estimating the moving velocity of the train and the path loss with the exploitation of pilots placed inside the frame. Then a joint algorithm for MAP estimation of path loss and ML Estimation for DFOs is designed based on the moving velocity of the train. We also proposed a joint detection algorithm in receiver to exploit multi-antenna diversity gains. The theoretical CRB for the joint channel estimation and DFO estimation is also derived. Simulation results shows low DFO estimation error and BER, and the simulation performance approaches the theoretical lower bounds, especially when the $k$ is large $(k>5)$. 


\section{Acknowledgments}

This work was partly supported by the China Major State Basic Research Development Program (973 Program, No. 2012CB316100), National Natural Science Foundation of China (No. 61171064), the China National Science and Technology Major Project (No. 2010ZX03003-003), NSFC (No. 61021001) and the Open Research Fund of National Mobile Communications Research Laboratory, Southeast University (No. 2011D13).

\section{References}

[1] J.Z. Wang, H.L. Zhu, N.J. Gomes, Distributed antenna systems for mobile communications in high speed Trains, IEEE Journal on Selected Areas in Communications, 2012, 30(4): 675-683.

[2] D.T. Fokum, V.S. Frost, A survey on methods for broadband internet access on trains, IEEE Communications Surveys \& Tutorials, 2010, 12(2): 171-185.

[3] K. Guan, Z.D. Zhong, B. Ai, Assessment of LTE-R using high speed railway channel model, In: Proc. 3rd International Conference on Communications and Mobile Computing, Qingdao, 2011: 461-464.

[4] L. Liu, C. Tao, J.H. Qiu, et al., Position-based modeling for wireless channel on high-speed railway under a viaduct at $2.35 \mathrm{GHz}$, IEEE Journal on Selected Areas in Communications, 2012, 30(4): 834-845.
[5] Y.Q. Zhou, F. Adachi, X.D. Wang, et al., Broadband wireless communications for high speed vehicles, IEEE Journal on Selected Areas in Communications, 2012, 30(4): 673-674.

[6] L.H. Yang, G.L. Ren, Z.L. Qiu, A novel Doppler frequency offset estimation method for DVB-T system in HST environment, IEEE Transactions on Broadcasting, 2012, 58(1): 139-143.

[7] E.P. Simon, L. Ros, H. Hijazi, et al., Joint carrier frequency offset and channel estimation for OFDM systems via the EM algorithm in the presence of very high mobility, IEEE Transactions on Signal Processing, 2012, 60(2): 754-765.

[8] A.G. Zajic, Estimation of mobile velocities and direction of movement in mobile-to-mobile wireless fading channels, IEEE Transactions on Vehicular Technology, 2012, 61(1): 130-139.

[9] Y.H.R. Zheng, C.S. Xiao, Mobile speed estimation for broadband wireless communications over Rician fading channels, IEEE Transactions on Wireless Communications, 2009, 8(1): 1-5.

[10] C.H. Tepedelenlioglu, G.B. Giannakis, On velocity estimation and correlation properties of narrow-band mobile communication channels, IEEE Transactions on Vehicular Technology, 2001, 50(4): 1039-1052.

[11] D. Tse, P. Viswanath, Fundamentals of Wireless Communication, Cambridge: Cambridge University Press, 2005.

[12] J. Proakis, M. Salehi, Digital Communications, New York: The McGraw-Hill Companies, 2000.

(Editor: Yao ZHOU) 